\title{
Editorial. Les jeux du politique et de la transnationalité
}

Didier Bigo

\section{(2) OpenEdition \\ 12 Journals}

Édition électronique

URL : http://journals.openedition.org/conflits/760

DOI : $10.4000 /$ conflits.760

ISSN : $1777-5345$

Éditeur :

CCLS - Centre d'études sur les conflits lilberté et sécurité, L'Harmattan

Édition imprimée

Date de publication : 21 mai 1992

ISSN : 1157-996X

Référence électronique

Didier Bigo, «Editorial. Les jeux du politique et de la transnationalité », Cultures \& Conflits [En ligne], 05 | printemps 1992, mis en ligne le 07 janvier 2003, consulté le 30 mars 2021. URL : http://

journals.openedition.org/conflits/760 ; DOI : https://doi.org/10.4000/conflits.760

Ce document a été généré automatiquement le 30 mars 2021.

Creative Commons License 


\title{
Editorial. Les jeux du politique et de la transnationalité
}

\author{
Didier Bigo
}

Les grands mouvements de contestation qui ont touché quasi-simultanément l'Europe centrale et orientale puis l'URSS, l'Afrique subsaharienne et l'Afrique australe, sont venus s'ajouter à ceux que connaissaient déjà l'Amérique latine, le sous-continent indien et le Maghreb-Machreq. Les années 1989, 1990 et 1991 auront donc été celles de la multiplication à l'échelle mondiale des contestations populaires ouvertes contre des pouvoirs en mal de légitimité ${ }^{1}$.

Les événements de 1989 obligent à se poser la question de la simultanéité des "vagues" de contestation et à ne pas envisager ces dernières uniquement comme des séries de coïncidences ou même, comme des réactions en chaîne des politiques d'états, après l'effondrement soviétique et les transformations géopolitiques subséquentes. Il semble que le contexte mondial où la transnationalisation joue à plein, où la médiatisation réduit les temps de réaction et favorise les mécanismes mimétiques, produise certains effets analysables en terme de stratégies d'acteurs et de contraintes structurelles supplémentaires dépassant le cadre national et s'imposant aux contestataires comme aux dirigeants. L'ambition de ce numéro est d'essayer d'apporter quelques éclaircissements sur ce phénomène crucial de la transnationalisation de la contestation.

La transnationalisation de la contestation : formulation d'une hypothèse

Certes, il n'y a pas rupture complète avec la période précédente. Depuis longtemps on connaît des exemples de contestations à l'échelon mondial, que ce soit dans les années 1848/1850, dans les années 1920 à la suite de la Première Guerre mondiale, dans les années 50 avec les premières décolonisations, dans les années 60 avec la contestation noire américaine et les contestations étudiantes, mais il semble que ces contestations populaires étaient relativement autonomes, segmentées, et étaient prioritairement structurées par le contexte politique national. Or, maintenant, si les cadres étatiquesnationaux jouent toujours un rôle important, si les gouvernements s'activent sur les plans diplomatique, économique et militaire, ils sont sérieusement concurrencés par 
d'autres acteurs: firmes multinationales, minorités ethniques, diasporas, flux d'immigrants et de réfugiés, autorités régionales, bureaucraties autonomisées par rapport au pouvoir central, internationales de partis, organisations transnationales ${ }^{2}$

Tous ces nouveaux pouvoirs engendrent aussi des formes particulières de contestation et tendent à rendre le monde contemporain tout à la fois plus interdépendant mais avec des acteurs plus autonomes et moins soumis aux autorités étatiques. James Rosenau oppose à cet égard le vieux monde dominé par les états

(state-centric) au nouveau plus complexe et plus multiforme (multi-centric) ${ }^{3}$. Comme il le rappelle, ces transformations de structures ne se sont pas faites en un jour, 1989 n'est guère qu'un révélateur des changements en cours. La date n'a rien de "magique" et elle n'est que l'aboutissement de processus engagés pour certains dès les années 1960/70, voire depuis le début du siècle. Mais ces transformations sous-jacentes ont toujours été niées ou marginalisées par la science politique internationaliste qui se focalise sur les états. Elle l'a payé d'une myopie sur les événements d'Europe centrale et sur les transformations actue11es du nationalisme. L'aveuglement est même trop systématique pour qu'il s'agisse d'ignorance. Il y a là une méconnaissance produite par l'attachement de la discipline aux instances gouvernementales et à sa volonté permanente de conseiller du prince. Les réactions et les analyses de la guerre du Golfe confortent ce diagnostic. Les discours officiels et nombre de discours académiques évacuent les dimensions transnationales et veulent n'y voir qu'une guerre très classique où les seules nouveautés seraient technologiques. L'occultation quasi délibérée du transnational, qu'il passe par les vecteurs de la croyance religieuse, des diasporas, des mouvements de solidarité provoque alors de nouvelles tensions politiques qui ne peuvent être régulées que si elles sont reconnues. Les analyses du cas indien par Christophe Jaffrelot, de la contestation en pays islamique par Bernard Botiveau, de l'Afrique du Sud par Philippe Chapleau et de l'ex-Yougoslavie par Natacha Rajakovic montrent toute l'importance de ces facteurs transnationaux dans l'analyse des processus de contestation.

Comprenons-nous bien : il n'y a pas rupture historique, il y a plutôt reconnaissance d'un nouveau paradigme. La lecture par les structures d'opportunité nationale de la contestation, si elle est toujours en partie pertinente, souffre de ne pas reconnaître la dimension transnationale, et plus elle tarde à le faire, plus cette dernière ayant de l'influence, il en résultera des analyses des processus contestataires de plus en plus pauvres, que ce soit à l'échelle macro ou micro. S'il fallait donner quelques exemples de modalités d'actions transnationales qui s'inscrivent dans les répertoires de contestation de nombreux mouvements, on peut en trouver dès les années 1950, et surtout à partir du milieu des années 1970 au moment où la diaspora palestinienne innove en utilisant de nouveaux répertoires d'actions comme les détournements d'avions ou les attentats fortement médiatisés créant cette forme originale d'action que l'on labellisera sous le terme erroné de "terrorisme international". On voit donc apparaître des pratiques de contestation qui s'insèrent dans un monde fortement internationalisé et médiatisé et en utilisent les ressources dès les années 1970. Avec les années 1980 et le début des années 1990, cette tendance des acteurs à jouer du transnational et à reprendre des pratiques inventées ailleurs se renforce ${ }^{4}$. La scène politique nationale n'est plus forcément la structure d'opportunité politique dominante. 
Selon les problèmes, il s'agit immédiatement de l'ensemble planétaire (écologie) ou beaucoup plus fréquemment de sous-ensembles régionaux (monde musulman, "bloc de l'Est", CEE...) ou encore de sous-ensembles n'épousant pas de frontières territoriales étatiques (primordialisme, radicalisme religieux, diasporas). De plus, des interconnexions apparaissent sans qu'un quelconque lien idéologique ou culturel ne soit apparent.

Seulement, comme le souligne Charles Tilly dans son texte, le lien direct supposé entre les nouveaux modes d'action du processus contestataire avec la médiatisation, les facilités de transport, les autres transformations de l'après-guerre qui expliquerait l'efficacité, la rapidité et la quasi-simultanéité des contestations par un effet de diffusion dû à la vitesse d'information dans le "village planétaire", fait office trop souvent d'analyse et déconsidère en partie la problématique transnationale. Ceci tient pour beaucoup aux confusions entre mondialisation, internationalisation et transnationalisation. Le premier terme est souvent synonyme dans la pensée politique d'une vision hégélienne de l'Etat et d'une homogénéisation progressive des comportements sous l'effet de la mondialisation, confondue avec l'occidentalisation : les discours sur la démocratie de marché et son extension mondiale en sont une caricature tout comme ceux où les médias hypostasient leur propre pouvoir et se mettent eux-mêmes en scène en s'auto-interrogeant et en se croyant le centre de tout ce qui arrive. L'internationalisation montre à quel point les politiques nationales perdent de leur autonomie et dépendent du contexte géopolitique et économique global, en revanche elles se situent toujours au niveau des politiques d'état. La transnationalisation met au contraire l'accent sur les acteurs libres de souveraineté, sur leur identité non territorialisée selon les frontières d'état, et sur la globalisation que cela implique mais qui va de pair avec une très grande différenciation. Le parallèle entre 1989 et 1848 a peut-être valeur heuristique pour mettre en évidence ces distinctions. En effet, dès 1848 , on avait vu des répertoires de contestation se transmettrent d'un pays à l'autre, en innovant justement par rapport aux anciens répertoires et ce, alors que les conditions de la médiatisation étaient encore très rudimentaires. Charles Tilly fait des années 1850 une période charnière où les innovations en matière d'actions collectives vont transformer le répertoire de contestation encore très communautaire, patronné et local en un répertoire plus associatif, plus autonome et plus national ${ }^{5}$. S'il y a transmission des modes d'action, c'est que ces innovations ne seraient pas restées inaperçues et auraient été reprises par d'autres groupes sociaux dans des contextes politiques différents, avec plus ou moins de succès. Les formes de répression comme les nouvelles transactions envisageables se seraient apprises aussi rapidement (voire plus) entre pouvoirs, que les nouvelles formes de contestation entre mouvements sociaux. La presse aurait certes joué un rôle quant à l'information initiale, mais l'essentiel serait dans le changement des anticipations des acteurs collectifs quant à l'efficacité des modalités d'action et quant à leur rapport de force. La transmission d'un savoir pratique entre acteurs qui n'agissent pas au sein de la même structure d'opportunité politique serait ainsi possible même si les innovations, en étant reprises, se moulaient sur les formes historiques traditionnelles du pays. Anthony Oberschall fait quasiment la même analyse de 1848 en insistant sur l'analyse du point focal qu'aurait été Paris à cette époque ${ }^{6}$. Il y a donc des mécanismes de transmission internationale des répertoires de contestation qui existent, avec d'ailleurs un avantage dans la rapidité de diffusion des modalités d'action des dirigeants (répression, transaction, nouvelles techniques) sur celles des contestataires et qui 
passent par des vecteurs qui n'ont guère de rapport avec la médiatisation et la rapidité des transports.

On voit donc que le rôle de l'international, ou mieux du transnational, est ancien mais il a toujours été considéré dans les analyses comme facteur secondaire ou permissif, non comme un facteur déterminant. Or, progressivement, n'est-il pas en train de concurrencer sérieusement les explications par le seul contexte politique national ? $\mathrm{N}$ 'assistons-nous pas à une mutation des répertoires d'action aussi considérable que celle qui a eu lieu au cours des années 1850 ? Mutation commencée sans doute dès les années 70 mais qui ne prend toute son ampleur que récemment avec la reprise de contestations populaires massives profitant de la délégitimation momentanée de l'usage d'une répression tout aussi massive des opposants par des moyens militaires classiques. 1989 serait alors, au même titre que 1848, le révélateur de transformations significatives dans les modalités de la contestation avec le passage, cette fois, d'un répertoire associatif, national, autonome à un répertoire local ou communautaire, transnational et interdépendant ${ }^{7}$.

Nous voudrions tester ici cette hypothèse qui, à notre avis, présente un double avantage : premièrement relativiser très fortement les approches géo-démographiques sur l'urbanisme dans le tiers-monde ou les approches économicistes sous leur double volet émeutes de la faim, émeutes du FMI, en redonnant à l'explication par le politique le rôle central qui est le sien; deuxièmement mettre l'accent sur l'intérêt d'une approche qui, en refusant le clivage disciplinaire science politique interne et internationaliste, soit à même de relier les hypothèses les plus fortes de l'analyse des mouvements sociaux que l'on trouve chez Charles Tilly, avec celles de l'analyse des émeutes par A Oberschall et avec celles de l'analyse du nouvel ordre mondial transnational que l'on trouve chez James Rosenau.

Retour au politique : l'abandon de l'économicisme

Jusqu'à présent, les seules explications "mondialisées", met tant l'accent sur des formes ou des causalités communes à des émeutes ayant lieu en Amérique latine, en Inde, en Afrique subsaharienne l'ont été par des approches qui tentaient de définir les caractéristiques des mobilisations émeutières de l'extérieur en mettant l'accent sur leur morphologie propre et leur localisation. La notion d'émeute urbaine, inorganisée, violente, avec des pillages, qui s'impose comme la plus spontanée dans notre imaginaire, provient des nombreux discours sur la ville dans le tiers-monde prise comme symbole de l'échec d'un développement harmonieux ${ }^{8}$. Géographes, urbanistes, économistes, organisations internationales font de la ville du tiers-monde un nouveau monstre. Des faubourgs des grandes villes du tiers-monde (mais aussi peut-être du Nord) émerge une nouvelle classe dangereuse menaçant les privilégiés. Cette "classe", cette "Nouvelle plèbe frumentaire" se constitue en homogénéisant dans une sousculture de la pauvreté les déracinés et les exclus de toutes sortes ${ }^{9}$. Ces exclus ne pourraient heureusement ou malheureusement, se faire entendre que rarement, lorsque la violence interne qui caractérise leurs rapports se catalyserait contre le responsable supposé de leurs difficultés. Véritable "chaudron", le moindre incident pourrait être le déclencheur de violences : une augmentation du prix de la farine, une hausse de loyer, un non-paiement des salaires des rares fonctionnaires qui font vivre les familles, une modification de la législation religieuse Imprévisibles, ces émeutes n'en auraient pas moins des causes structurelles. Emeutes du pain, émeutes de la faim auraient à leur origine une dégradation des conditions économiques des populations suite aux difficultés de s'adapter à une structure démographique à forte natalité 
entraînant un exode rural et une implosion urbaine, débouchant sur une augmentation des frustrations relatives de populations qui verraient leur minimum vital atteint au moment où les images du monde leur proposent des modèles de consommation largement occidentalisés ${ }^{10}$. Cette explication économiciste influencée par les thèses de Neil Smelser, Ted Gurr et Harry Eckstein continue d'alimenter les médias occidentaux". Mettant en avant les caractéristiques non rationnelles de la mobilisation des foules et la spontanéité des comportements récupérés après coup par des organisations politiques, le modèle tend à rejeter l'analyse de la dynamique de la mobilisation émeutière et de son rapport au politique pour lui substituer en amont une question sur les causes structurelles des émeutes et en aval une sur la traduction en langage politique de telles émeutes par certains mouvements d'opposition. Il en résulte souvent une coupure qui néglige ce qui se passe dans l'émeute dans la contestation ${ }^{11}$. On suppose que les acteurs de l'émeute ne sont pas en continuité avec ceux qui émettent les revendications politiques et qu'il existe en quelque sorte deux scènes : la scène de la contestation des "déshérités", assez radicale mais sans projet et la scène politique où pouvoir et groupes divers d'opposants cherchent à donner à l'émeute une signification politique qui légitime leur existence. Dans l'espace maghrébin, souvent donné en exemple (Tunisie, Maroc, Algérie), les émeutiers, souvent des jeunes, se seraient mobilisés sur des motifs très disparates, dénonciation de la faim, mauvais fonctionnement du système scolaire, corruption, rôle de l'étranger que la nature des pillages rendrait plus explicite encore que les slogans. Cela expliquerait aussi la faible durée des contestations et le fait que le pouvoir puisse parfois habilement récupérer les émeutiers après quelques concessions de façade. La fluidité de la mobilisation, la grande rotation des individus engagés dans les journées d'émeutes successives rendraient malgré tout celles-ci imprévisibles et déstabiliseraient de temps en temps le pouvoir qui chercherait à faire émerger des leaders pour prendre contact ou/et réprimer. Devant la "spontanéité" des émeutes, il y aurait alors parfois complicité objective entre le pouvoir et des groupes d'opposants, le premier cherchant des responsables et les seconds visant à se faire reconnaître comme les instigateurs de telles mobilisations. Par exemple, nombre de groupes radicaux au Maghreb auraient cherché à profiter d'émeutes de ce type pour mobiliser les foules contre le pouvoir en place et contre les valeurs occidentales rendues responsables des maux de la société ${ }^{12}$. On pourrait analyser quasiment de la même manière ce qui se passe en Corée, en Afrique du Sud, en Amérique latine, au Nigeria, en Inde... Partout les banlieues, l'inactivité de la jeunesse, les mauvaises conditions de vie seraient les facteurs décisifs du passage à la contestation émeutière. Mobilisés pour des raisons religieuses ou économiques, ils formeraient les "bataillons" de groupuscules politiques qui viseraient à transformer le sens de la manifestation. La contestation serait en outre renforcée par les répressions souvent disproportionnées avec les dégâts provoqués par les émeutiers.

Une autre variante plus politique, mais finalement peu différente de la première dans son économicisme, verrait dans ces émeutes, le résultat des politiques du FMI et de la Banque mondiale, c'est-à-dire, pour cette approche dépendantiste, le résultat des politiques ultra-libérales du reaganisme triomphant fondamentalement inadaptées au tiers-monde. Les "émeutes FMI" viendraient d'une nouvelle phase de l'hégémonie capitaliste mondiale et des tentatives populaires d'échapper à cette nouvelle division $\mathrm{du}$ travail. Elles seraient une réaction des groupes vulnérables aux politiques d'ajustement passant non par une remise en cause explicite de la théorie économique du FMI mais par les conséquences directes de l'ajustement (hausse des prix de la farine, 
de l'essence). Souvent très marquée par l'analyse marxisante, cette thèse met l'accent sur les contradictions structurelles de l'économie dans les pays du tiers-monde et est particulièrement en vigueur pour expliquer les émeutes du continent latino-américain : Caracas, Buenos Aires ou d'Afrique subsaharienne : Abidjan, Libreville". . Le FMI et la Banque mondiale, par le biais de la conditionnalité économique et politique, gouverneraient de plus en plus par procuration les pays du Sud où il est encore utile de rester et abandonneraient les autres. Négligeant fortement les attentes des populations à l'égard de certains programmes de la Banque et le jeu complexe entre la Banque, les pouvoirs en place et les populations urbaines et rurales, ces analyses souffrent pour la plupart d'un manichéisme certain. Souvent les émeutes proviennent de catégories auparavant privilégiées (fonctionnaires, étudiants), les campagnes étant peu hostiles à de nouvelles répartitions de revenus ; les émeutes sont aussi parfois instrumentalisées par les acteurs dominants en vue de susciter une réaction "nationaliste" contre l'étranger De plus les corrélations attendues entre émeute urbaine et intensité du programme d'ajustement structurel ne se retrouvent absolument pas. Enfin, méthodologiquement, peut-on inférer de causes structurelles très générales, l'ici et le maintenant des contestations? N'y a-t-il pas là une illusion étiologique qui occulte la dynamique même des jeux politiques internes à la contestation populaire?

Dans ces différents cas, l'explication par le politique, les conduites rationnelles des acteurs sociaux contestataires sont pour le moins minorées (première variante) ou subordonnées à des lois de l'histoire (seconde variante). Certes elles apparaissent mais semblent subordonnées à des contraintes structurelles ou psychosociologiques telles qu'elles ne peuvent guère expliquer que les infimes variations des expériences concrètes et ne sont pas intégrées comme éléments explicatifs du modèle de l'émeute urbaine. En supposant par exemple dans la première variante une coupure entre les populations émeutières et les membres politiques gérant la contestation, ces analyses esquivent la question de l'organisation de la contestation, du rapport entre les "quasiprofessionnels" du politique contestataire et l'ensemble des acteurs qui se mobilisent (y compris bien sûr les groupes dirigeants). Elles font comme si l'organisation partisane, les symboles identitaires fabriqués par les propagandes religieuses et politiques n'avaient pas d'importance. Elles négligent les luttes symboliques et les capacités d'auto-organisation, d'invention du politique par la contestation populaìrel5. Elles n'analysent guère les formes prises par l'émeute, son rapport aux autres répertoires d'action et la fréquente ritualisation qui entoure ces formes d'actions soi-disant spontanées. Elles ignorent en outre les dynamiques transnationales de mobilisation et les effets proprement politiques du contexte mondial. Or, toutes les analyses détaillées montrent à quel point ces traits sont déterminants. Christophe Jaffrelot montre par exemple comment les symboles identitaires des communautés musulmanes et hindous sont déterminants dans la mobilisation et que les formes processionnelles qui sont utilisées, renforcent l'unité du groupe et sa croyance d'être une seule et même entité. Philippe Chapleau rappelle comment les groupes d'Inkatha calquent les "impi" traditionnels pour mobiliser leurs troupes. Yves Botiveau montre les stratégies des groupes islamistes et leur capacité d'organiser la mobilisation des foules. Christophe Jaffrelot montre aussi à quel point la diaspora musulmane en Inde joue du contexte international pour changer de profil et émettre des exigences politiques que son statut et sa place démographique ne lui permettent guère sur le plan interne. Elle finirait même par créer en effet de retour, chez les hindous, un complexe d'infériorité majoritaire qui aurait relancé les mouvements primordialistes. Il montre enfin à quel 
point les jeux politiques sont bien plus déterminants que les motivations économiques pour comprendre les émeutes indiennes rejoignant une fois encore Philippe Chapleau lorsqu'il décrit la stratégie Buthelezi ou Bernard Botiveau à propos des islamistes.

Bref, dans les thèses "urbanistes", la mondialisation est du ressort de l'économique, du démographique, pas du politique ou du culturel. L'avantage de ces modélisations est sans doute de permettre de penser de nombreux phénomènes autrement que par la simple réduplication accidentelle de cas de figures différents et dont les similitudes seraient simplement superficielles. Ces modélisations ont tendance, en ignorant le politique, à le réintroduire subrepticement par la désignation de catégories sociales "fautives", responsables des comportements de violence, elles ne sont donc pas capables de penser le transnational. Pour elles, mondialisation va de pair avec homogénéisation, uniformisation. Elles sont donc à l'opposé de la problématique du numéro où tous les auteurs se retrouvent pour montrer que la globalisation transnationale va de pair avec la différenciation locale, que ce soit chez Bernard Botiveau à travers les différents cas de figures de la contestation islamique ou chez Natacha Rajakovic à propos du yougoslavisme.

Le risque, en abandonnant ces visions économicistes, serait d'abandonner le projet comparatif et l'explication des interdépendances entre les phénomènes. C'est souvent le travers dans lequel tombent les approches monographiques par l'histoire ou la sociologie culturelle, qui si elles mettent l'accent sur l'irréductibilité des trajectoires historiques spécifiques, répugnent au comparatisme systématique et versent parfois dans un culturalisme qui est à l'opposé des véritables analyses sur les dynamiques culturelles. Très souvent les auteurs insistent sur la spécificité de tel ou tel cas de figure et refusent de le penser dans sa coexistence avec d'autres cas partout similaires qui ont pourtant pu l'influencer. Aussi importantes soient-elles ces monographies souffrent donc d'une lacune: sensibles aux facteurs politiques internes, locaux ou nationaux, elles ont souvent tendance à négliger les facteurs transnationaux et mondiaux. Le rôle du contexte international, de la situation mondiale n'est évoqué qu'à titre de "paysage" et non comme variable explicative. Quelques thèses ont même tendance à renvoyer l'émeute à des caractéristiques particulières des populations : antagonismes historiques entre groupes ethniques, souvent analysés comme des entités fixes et transhistoriques, niveau de violence existant dans la société Les protestations actuelles d'Europe centrale contre le pouvoir ou certaines communautés sont déjà lues comme le retour aux "vieux démons", comme si la haine de populations soi-disant ethniquement différentes permettait de comprendre et d'expliquer les violences. Natacha Rajakovic sur le cas yougoslave montre justement à quel point ces visions sont éloignées des pratiques sociales des acteurs et de leurs motivations. Philippe Chapleau récuse la thèse ethnique des black on black violence. Christophe Jaffrelot montre à quel point les constructions identitaires sont historicisées. En effet les identités sont toujours fluctuantes et instrumentalisées par des entrepreneurs politiques à des fins de mobilisation. Elles se chevauchent et n'ont jamais le caractère essentialiste qu'on leur prête souvent. Ainsi, le retour à l'explication par le politique est essentielle, elle permet de comprendre les enjeux des émeutes en évitant les explications par l'urbain, l'économique ou l'ethnique. L'explication par le politique doit être combinée avec la réflexion sur la transnationalité pour éviter de perdre de vue la simultanéité de certains processus.

Vers des modalités de contestations transnationales Seuls quelques travaux ont déjà lancé des pistes de recherche sur le thème des transformations des répertoires de contestation, soit en travaillant sur longue périodepour mettre au jour les mutations 
(souvent assez faibles selon C. Tilly) des répertoires d'action collective ${ }^{13}$ dans les sociétés européennes, soit en comparant comme A. Oberschall différentes modalités de contestation en les distinguant en fonction des liens au sein des collectivités et entre les collectivités, selon qu'ils sont communautaires, inorganisés ou associatifs d'une part, segmentés ou intégrés d'autre part ${ }^{14}$. Cette deuxième approche, en définissant plus ou moins des aires de pertinence culturelle, permettrait par exemple de parler d'une culture de l'émeute au sens strict dans le monde musulman et particulièrement dans le Maghreb-Machreq où le système de représentation et de légitimation du pouvoir fragilise l'état et le laisse démuni face à une contestation qui en appelle au répertoire du religieux, alors qu'en Occident ou en Afrique subsaharienne les contestations populaires seraient beaucoup plus des formes de contestations demandes (et non des contestations-rejets comme l'émeute) en appelant à l'état pour la satisfaction de besoins particuliers ce qui inévitablement renforcerait celui-ci ${ }^{15}$. Elle a l'avantage de montrer contrairement à ce que supposait les thèses sur la subculture de la pauvreté dans les bidonvilles, que les allégeances communautaires ne disparaissent pas en ville, qu'elles sont adaptées, retraduites comme les travaux des anthropologues (Balandier ou Jean-Loup Amselle) l'ont largement prouvé. Elle permet aussi, en insistant sur les réseaux de solidarité et de clientélisme, de prendre en défaut la coupure : ville/rural. Elle permet enfin d'articuler clairement le rapport au politique et ses différentes expressions dans les sociétés. Mais la pertinence de la distinction entre l'émeute contestation-rejet, inorganisée et segmentée et les autres formes de contestation reste problématique surtout si on renvoie les distinctions typologiques à des formes civilisationnelles particulières. De nombreux auteurs travaillant sur le thème ont avancé, que ce soit pour les émeutes de Kano au Nigeria, les émeutes en Tunisie et en Algérie, les émeutes du Soudan et d'égypte que le rejet de l'état était quelque chose de puissant, mais peut-on parler pour autant de contestation "hors système", d'expression "libre" ? N'y a-t-il pas un risque de reprendre la coupure entre les scènes de la contestation et du politique au lieu de tisser les liens entre les acteurs et les leaders émergents? Ne doit-on pas nuancer aussi les catégories d'Oberschall et l'attachement de l'émeute au communautaire et à l'inorganisé face à la manifestation et à l'associatif ? N'y a-t-il pas un danger de reconduction des thèses sur l'irrationnel émeutier face au rationnel manifestant, et de reprendre le dualisme émeutiers, dirigeants du mouvement? Peut-on véritablement distinguer des zones réservées aux manifestations et des zones dans lesquelles les émeutes seraient plus fréquentes que dans d'autres lieux? Monde musulman et sous-continent indien seraient-ils vraiment plus touchés que l'Afrique australe, l'Afrique subsaharienne, l'Amérique latine ou l'Extrême-Orient? L'Europe occidentale serait-elle épargnée en ne connaissant plus qu'une forme manifestante euphémisant la violence? Derrière ces questions s'en profile une plus fondamentale encore. Peut-on lier les approches de sociologie comparée dans la lignée d'Oberschall, les travaux plus historiques de Charles Tilly sur l'Europe et les questions sur le nouvel ordre international ? Comment faire pour penser les émeutes urbaines, non de manière essentialiste ou typologique, mais comme une forme particulière de contestation populaire ouverte qui fait souvent l'objet de transformation dynamique dans son rapport aux formes de violence utilisées, dans son rapport aux acteurs initiaux et dans son rapport au transnational ?

Que ce soit par aire culturelle ou/et par champ politique national, le primat souvent mis sur la structuration des opportunités politiques dans ce contexte défini, laisse peu de place en général à la dimension globale proprement dite. Celle-ci n'est pas intégrée à 
l'analyse. Par exemple, les travaux de Marco Giugni et Hanspeter Kriesi ont montré, à partir d'études sur les mouvements sociaux en Suisse, que les thèmes internationaux, lorsqu'ils existent, ne sont pas déterminants dans la mobilisation parce qu'ils souffrent d'un manque de coordination. Selon eux, les mouvements sociaux se sont jusqu'à présent typiquement mobilisés dans le cadre des politiques nationales, parce qu'historiquement constitués au dix-neuvième, ils en garderaient la structuration nationale et parce que les nouveaux mouvements sociaux seraient plus tournés vers le local que vers le national ou le transnational. Aussi pertinente soit leur étude sur la sensibilité des acteurs collectifs à la forme de l'état et au contexte politique, comme ils envisagent la globalisation uniquement sous l'angle du supranational et d'une coordination internationale, ils ont tendance à restreindre fortement la portée de leur analyse et laissent échapper la question du transnational tant comme sensibilité des acteurs libres de souveraineté à des événements, des contextes qui dépassent l'étatnation, qu'en tant que facteur de structuration identitaire et de champ d'énonciation. Si au contraire, on élargit la question des mouvements sociaux à celle des processus de contestation, y compris hors de la sphère de l'Europe occidentale et en y incluant aussi les émeutes urbaines, on aura un autre regard sur le sujet. La dimension globale ne peut être ramenée à un pur et simple effet de diffusion, d'importation de modèles (d'idéologies, de techniques, de comportements, d'actions, de relations) de groupe social à groupe social, de dirigeants à dirigeants. Elle n'est pas non plus réductible à la seule interdépendance de contextes politiques. Les effets de mondialisation communicationnelle et d'internationalisation jouent certainement mais la globalisation passe avant tout par la transnationalisation et la nouvelle forme "d'ordre international turbulent" qu'elle implique. A l'époque contemporaine, aucun phénomène n'est véritablement indépendant et sans effet sur les autres. Au contraire, tous sont reliés, mis en réseau, ce qui implique des interactions réciproques très difficiles à évaluer mais qui sont au coeur des dynamiques sociopolitiques. La contingence, le hasard ne sont plus simplement des résidus de l'analyse comme dans les premiers modèles mais en deviennent le centre quand on comprend la manière dont ils sont des principes créateurs qui, semble-t-il, jouent à la fois dans le sens d'une plus grande globalisation par renforcement des interconnexions et d'une plus grande différenciation et complexité interne à l'échelle micro, locale. L'analyse de la transnationalité comme niveau mêlant le micro des situations locales et le macro des situations mondiales devient alors indispensable comme le suggère Rosenau.

C'est après tout ce que nous propose aussi Charles Tilly lorsqu'il insiste sur le "réseau des événements de 1989" et sur la notion de "cycle de contestation" de Sidney Tarrow ${ }^{16}$. C'est aussi la prolongation de l'hypothèse d'Oberschall sur les points de focalisation internationaux. Il est donc possible de repérer des points de convergence entre analyses pour l'instant fortement indépendantes suite au cloisonnement disciplinaire et que l'on pourrait relier afin de mieux éclairer les transformations des contestations contemporaines.

Si l'on reprend l'exemple des événements de 1989, qui partent du point focal de Moscou, ceci nous amène à les lire dans leur dynamique d'ensemble et à souligner le fait que leur contingence n'en structure pas moins fortement les comportements des acteurs collectifs contemporains - où que ce soit sur la planète - et ce, malgré la diversité des formes populaires de contestations qu'ils rencontrent et qui peuvent largement différer des processus d'Europe centrale. En ce sens les modalités contestataires ne sont pas uniquement la résultante des transformations géopolitiques 
impliquées par la désagrégation de l'empire soviétique. La métaphore des ondes de choc est impuissante à rendre compte de la diversité des contestations et de leur quasisimultanéité. Si les contestations indiennes, coréennes, ivoiriennes se produisent en 1989, elles ne sont pas la résultante de la chute du communisme mais ont pourtant des relations avec le contexte mondial. Ni sous le signe de la nécessité, ni sous le signe de l'indépendance, les événements initiaux ont des propriétés morphogénétiques qui donnent quelques unes des caractéristiques contemporaines de cet "ordre mondial relâché", de ce "chaos à îlots ordonnés", de ces "turbulences" qui ont succédé à la tendance structurelle à la bipolarisation que nous connaissions plus ou moins depuis la fin des années 1950. Ils ont impliqué, via la transnationalisation, non seulement une transformation du contexte global mais aussi des formes de l'énonciation des identités des acteurs collectifs.

Par exemple, avec le refus répété de Michael Gorbatchev d'envisager une intervention militaire soviétique en faveur des pouvoirs communistes d'Europe centrale, il s'est produit non seulement une transformation sensible du rapport de forces existant entre les groupes dominants et les groupes sociaux subordonnés (permettant à ceux-ci d'envisager une contestation populaire ouverte) mais de plus, cela a délégitimé le recours massif à la coercition contre des populations désarmées, manifestant pacifiquement leur désaccord. D'une certaine manière, la réaction internationale à la place Tian' An Men, en Chine, a renforcé cette croyance qu'il n'était plus possible pour un pouvoir en place de réprimer sa population en se référant au principe de la souveraineté nationale et de la non-ingérence dans les affaires intérieures d'un état. Cela a momentanément gêné les autres pouvoirs politiques confrontés à de telles formes de contestation au moment où cela renforçait, du côté des mouvements sociaux, la croyance dans l'efficacité de ce type de protestation pour faire tomber un pouvoir, fut-il autoritaire. Il est bien sûr délicat de parler d'une homogénéisation momentanée des comportements des dirigeants, brusquement pris dans un réseau de significations qui leur faisait perdre leur propre croyance dans l'efficacité de la répression. Mais plus des régimes tombaient sans oser lancer une répression de masse, plus se confortait la croyance que celle-ci était inutile ou dépassée. La gestion du sens se faisait en faveur des contestataires (lesquels surestimaient souvent leurs propres capacités et lançaient des actions qui en d'autres temps auraient été suicidaires) et divergeait du rapport de puissance. La modalité d'action d'une contestation populaire massive désarmée était vécue et gérée par les leaders comme allant de pair avec un combat pour la mise en place de formes d'exercice démocratique du pouvoir quand bien même les motivations initiales des acteurs locaux étaient, éloignées de cette préoccupation et répondaient plutôt à des mobilisations communautaires, locales, religieuses L'énonciation transnationale jouait le rôle de catalyseur d'identité et rentrait dans les stratégies d'acquisition de soutiens extérieurs dans le combat contre le pouvoir en place. Ainsi, si les cibles de certaines émeutes étaient beaucoup plus tournées contre $d$ autres communautés que contre le pouvoir en place, et étaient aussi beaucoup plus victimaires, cela n'empêchait pas un discours quasi-similaire tenu par les leaders et des anticipations des dirigeants quasi-identiques à celles que nous avons identifiées face aux mouvements de contestation populaire désarmés. Paradoxalement, pendant un moment le critère de l'utilisation de la violence ou non par les acteurs contestataires n'a plus été opératoire dans les systèmes de (dé)légitimation alors qu'il continuait de l'être pour les groupes dirigeants. 
L'exemple des contestations en Afrique subsaharienne est significatif Celles-ci ont leurs origines propres. Les contextes nationaux, l'autoritarisme plus ou moins fort du pouvoir ont joué dans le sens dune différenciation très nette de chaque cas de figure et il n'est donc pas question de croire qu'elles seraient la suite du "vent de liberté ayant soufflé à l'Est" et propagé en Afrique par les médias, comme on 1a trop écrit. En revanche, les étudier séparément ne fait as sens. Ce que l'on a appelé la démocratisation de l'Afrique a eu lieu au moment où les dirigeants africains ont cru qu'ils ne pouvaient plus agir de manière autoritaire pour contrer leurs opposants et qu'ils étaient obligés d'accepter des "conférences nationales" s'ils voulaient maintenir leur crédibilité internationale et les financements qui en dépendent. Les contestataires ont anticipé cette fragilité et s'ils ont osé affronter les ré mes ils n'ont pu y arriver qu en mobilisant les populations à la fois à travers les ressources de la mémoire historique et des griefs accumulés durant des années mais aussi en Faisant explicitement un parallèle entre leurs dirigeants, fussent-ils anticommunistes et les dirigeants des pays d'Europe centrale et orientale. Le rôle particulier du gouvernement français à l'égard de l'Afrique subsaharienne a aussi joué dans la mesure où un intermédiaire étatique disait faire sienne l'émergence de la contrainte, interdisant aux dirigeants les répressions massives. D'ailleurs, on notera que le relâchement français a favorisé à nouveau un recours aux ressources coercitives et que dès lors d'autres sont tentés de les utiliser à nouveau. On voit donc à travers cet exemple à quel point la globalisation transnationale est fragile, momentanée et susceptible de renversement de tendances, même relayée par des politiques d'état. Elle est le contraire d'une marche assurée de l'Histoire. Parler à cet égard de "temps mondial" peut avoir des qualités heuristiques : de nouvelles significations s'imposent à tous les acteurs politiques et modifient profondément leurs calculs politiques. Ils ne peuvent échapper à une réévaluation de leurs critères d'actions face à de tels bouleversements (ou turbulences) internationaux. La globalisation qui se met en place lie des formes de contestation pourtant très éloignées les unes des autres. Seulement ceci ne signifie pas homogénéisation des situations mais interdépendance qui favorise la différenciation loin de la bloquer. Le temps mondial rend compte de cette imposition momentanée et réversible de problématique légitime qui s'impose à tous comme la seule manière d'agir. Bien au-delà des discours sur l'idéologie libérale, ce serait dans ces modalités d'actions et de réactions que l'on retrouverait l'impact de la mondialisation. Mais rappelons-le, le temps mondial n'exclut pas les temps locaux, au contraire. Il permet quasiment une fragmentation à l'infini, une dynamique en réseau brisant les cadres étatiques nationaux ou les relativisant à l'extrême. Les cadres d'action politique sont alors locaux, communautaires, transnationaux tout autant que nationaux. Christophe Jaffrelot tout comme Bernard Botiveau le montrent de manière exemplaire dans leurs textes. Plus près de nous, Alain Touraine, à propos de l'immigration en France et de sa vraie ou fausse intégration, montre le lien entre la globalisation et la différenciation qui s'accentuent ensemble en perturbant les politiques nationales. "Beaucoup plus largement, l'expérience de chacun a cessé d'être avant tout nationale. Partout dans le monde se séparent de plus en plus des flux transnationaux de biens économiques et culturels et des identifications de plus en plus proches, à une communauté, à une région, à un groupe défini, à une religion, une nation, un genre de vie. Nos sociétés enrichies ne sont pas de plus en plus homogènes; sous des formes différentes de celles des pays pauvres, elles se dualisent. C'est très visible aux Etats-Unis, où la misère côtoie 
la richesse et où se maintiennent ou même se durcissent des ghettos. Mais les mêmes tendances sont visibles en France comme en Grande-Bretagne ou en Allemagne" ${ }^{17}$.

$C^{\prime}$ 'est cet enchevêtrement des identifications plurielles perturbant la suprématie du cadre politique national en le poussant à la différenciation comme à la globalisation qu'il faut approfondir pour comprendre les nouvelles formes de contestation que l'on connaît actuellement. Ce numéro s'en fait l'écho à propos d'exemples pris hors d'Europe occidentale mais le prochain qui sera consacré aux violences urbaines dans les banlieues reviendra lui aussi sur ces questions.

\section{NOTES}

1. Pour une information chiffrée, cf. Approches polémologiques : conflits et violence politique au tournant des années $1990, \mathrm{FEDN}, 1991$. Près de $25 \%$ des actes recensés dans la presse à propos de la violence ressortent de mouvements de foule et de la répression qui s'abat sur eux. Auparavant la proportion était d'environ $15 \%$.

2. Pour une illustration concernant l'Etat par excellence, voir notre dernier numéro sur les Transferts d'armement et leur patrimonialisation par des bureaucraties, en Chine, en France, aux états Unis et encore plus tragiquement si l'on en croit la presse, dans l'ex-URSS : Les réseaux internationaux de violence, Cultures \& Conflits (4), hiver 1991. 3. James Rosenau, Turbulence in World Politics, Harvester Wheatsheaf 1990.

4. Acteurs étatiques, communautés, diasporas, groupes combattants se retrouvent dans les mêmes stratégies, malgré leur différence d'échelle que ce soit à propos d'attentats, de trafic de drogue, de trafic d'armes ou d'actions humanitaire

5. Charles Tilly, La France conteste de 1600 à nos jours, Paris, Fayard, 1986, p. 545.

6. Anthony Oberschall, Social Conflict \& Social Movements, Practice Hall, 1973, p. 139.

7. Local car les figures de l'identification collective se font à travers des réseaux de proximité qui empruntent les voies de l'ethnicité, du religieux ou de la territorialité à l'Echelle micro. Les idées nationales fédératrices, les projets étatiques, ne sont plus porteurs des mêmes dynamiques, ils sont traversés par la volonté d'autonomie des acteurs et se désagrègent facilement. Transnational car les flux de populations, de capitaux, de cultures, d'idéologies primordialistes se moquent des frontières et des contrôles étatiques toujours plus poreux. Interdépendant car ce qui se passe dans un pays affecte presque toujours ses voisins, voire le reste du monde lorsqu'il s'agit d'une ex grande puissance.

8. Voir la définition de l'émeute comme un soulèvement spontané ou non organisé pouvant prendre la forme d'un rassemblement accompagné de cris et de bagarres (Petit Robert).

9. Cf. Manières de voir 12 : la ville, recueil du Monde diplomatique ou J.C. Rufin, L'Empire et les nouveaux barbares, reprenant les thèses d'Oscar Lewis.

10. Yves Lacoste, "L'implosion urbaine", Hérodote (4), 1983, ainsi que plusieurs autres numéros de la revue Hérodote Gérard Chaliand, "Anthologie de la stratégie", 1990.

11. Michel Dobry "Calcul, concurrence et gestion du sens" in Pierre Favre, La Manifestation, Paris, Presses de la FNSP, 1990. 
12. Voir là encore la revue Hérodote, entre autre Karim Chergui, "Novembre 1986, la révolte des jeunes à Constantine", avril-juin 1987.

13. Charles Tilly, La France conteste de 1600 à nos jours, Paris, Fayard, 1986 ; Contrainte et capital dans la formation de l'Europe 990-1990, Paris, Aubier, 1992.

14. Anthony Oberschall, Social Conflict \& Social Movements, Practice Hall, 1973.

15. Bertrand Badie, Les deux Etats, Paris, Fayard, 1986 ; Badie, Hermet, Politique comparée, Paris, PUF, 1990.

16. Même si cette dernière pourrait avoir l'inconvénient de faire penser au cycle shumpétérien d'innovation, voire au cycle Tuglar donc à des effets de flux, d'apogée et de reflux quasi-naturels après certains événements particulièrement créateurs. En effet, rien ne prouve que les formes contestataires épousent cette logique des "vagues", des cycles ; elles peuvent être beaucoup plus aléatoires, dispersées et n'obéissent guère à cette linéarité cyclique. Il est difficile après tout de penser cycle et contingence simultanément.

17. Alain Touraine, "Vraie ou fausse intégration", Le Monde, 29.01.1992.

\section{RÉSUMÉS}

Les grands mouvements de contestation qui ont touché quasi-simultanément l'Europe centrale et orientale puis l'URSS, l'Afrique subsaharienne et l'Afrique australe, sont venus s'ajouter à ceux que connaissaient déjà l'Amérique latine, le sous-continent indien et le Maghreb-Machreq. Les années 1989, 1990 et 1991 auront donc été celles de la multiplication à l'échelle mondiale des contestations populaires ouvertes contre des pouvoirs en mal de légitimité.

Urban riots are often seen as the resultants of frustration caused by the deepening of economic crises or urban problems. Quite on the contrary, we would like to show the importance of political relations in the emergence of such violence. However, the " political explanation " is too often reduced to a " one country " analysis, thus failing to explain the globalisation and the simultaneity of riots, specially conspicuous during the 1989 events. Raising the question of the transnational transmission of the repertory of actions (contestation and coercion), we suggest that the delegitimisation, for a brief moment, of massive coercion by government partly explains how it was possible for a generalisation of protests to occur not only in central and eastern Europe but also in India, the Arab countries and French-speaking Africa..

\section{INDEX}

Mots-clés : sciences politiques, réseaux transnationaux 
AUTEUR

DIDIER BIGO

Maître de conférences des Universités à l'Institut d'Etudes Politiques de Paris, rédacteur en Chef de Cultures \& Conflits 